\title{
Regional microglia are transcriptionally distinct but similarly exacerbate neurodegeneration in a culture model of Parkinson's disease
}

\author{
Eric Wildon Kostuk', Jingli Cai ${ }^{1}$ and Lorraine lacovitti ${ }^{1,2,3^{*}}$ (D)
}

\begin{abstract}
Background: Parkinson's disease (PD) is characterized by selective degeneration of dopaminergic (DA) neurons of the substantia nigra pars compacta (SN) while neighboring ventral tegmental area (VTA) DA neurons are relatively spared. Mechanisms underlying the selective protection of the VTA and susceptibility of the SN are still mostly unknown. Here, we demonstrate the importance of balance between astrocytes and microglia in the susceptibility of SN DA neurons to the PD mimetic toxin 1-methyl-4-phenylpyridinium (MPP+).

Methods: Previously established methods were used to isolate astrocytes and microglia from the cortex (CTX), SN, and VTA, as well as embryonic midbrain DA neurons from the SN and VTA. The transcriptional profile of isolated microglia was examined for 21 canonical pro- and anti-inflammatory cytokines by qRT-PCR with and without MPP ${ }^{+}$ exposure. Homo- and heterotypic co-cultures of neurons and astrocytes were established, and the effect of altering the ratio of astrocytes and microglia in vitro on the susceptibility of midbrain DA neurons to the PD mimetic toxin $\mathrm{MPP}^{+}$was investigated.

Results: We found that regionally isolated microglia (SN, VTA, CTX) exhibit basal differences in their cytokine profiles and that activation of these microglia with $\mathrm{MPP}^{+}$results in differential cytokine upregulation. The addition of microglia to cultures of SN neurons and astrocytes was not sufficient to cause neurodegeneration; however, when challenged with $\mathrm{MPP}^{+}$, all regionally isolated microglia resulted in exacerbation of $\mathrm{MPP}^{+}$toxicity which was alleviated by inhibition of microglial activation. Furthermore, we demonstrated that isolated VTA, but not SN, astrocytes were able to mediate protection of both SN and VTA DA neurons even in the presence of exacerbatory microglia; however, this protection could be reversed by increasing the numbers of microglia present.

Conclusion: These results suggest that the balance of astrocytes and microglia within the midbrain is a key factor underlying the selective vulnerability of SN DA neurons seen in PD pathogenesis and that VTA astrocytes mediate protection of DA neurons which can be countered by greater numbers of deleterious microglia.
\end{abstract}

Keywords: Microglia, Astrocyte, Neuron, Parkinsons' disease, Neurodegeneration

\footnotetext{
* Correspondence: lorraine.iacovitti@jefferson.edu

'Department of Neuroscience, Farber Institute for Neurosciences, Thomas Jefferson University, Room 320, Bluemle Life Sciences Building, 233 S. 10th Street, Philadelphia, PA 19107, USA

${ }^{2}$ Department of Neurology, Farber Institute for Neurosciences, Thomas

Jefferson University, Room 320, Bluemle Life Sciences Building, 233 S. 10th

Street, Philadelphia, PA 19107, USA

Full list of author information is available at the end of the article
}

(c) The Author(s). 2018 Open Access This article is distributed under the terms of the Creative Commons Attribution 4.0 International License (http://creativecommons.org/licenses/by/4.0/), which permits unrestricted use, distribution, and reproduction in any medium, provided you give appropriate credit to the original author(s) and the source, provide a link to the Creative Commons license, and indicate if changes were made. The Creative Commons Public Domain Dedication waiver (http://creativecommons.org/publicdomain/zero/1.0/) applies to the data made available in this article, unless otherwise stated. 


\section{Background}

Parkinson's disease (PD) is a progressive neurodegenerative disease which is characterized by the selective degeneration of dopaminergic (DA) neurons of the substantia nigra pars compacta $(\mathrm{SN})$, whereas the neighboring DA neurons of the ventral tegmental area (VTA) are relatively spared [1]. Previous studies have examined the DA neurons of these two regions to better understand why only one subset of DA neurons is significantly more vulnerable to PD than the other [2-5]. Recently, greater focus has been placed on extrinsic mechanisms, such as the role of astrocytes, in the disease process suggesting that neuronal vulnerability results from both intrinsic and extrinsic influences. Indeed, our recent work (Kostuk et al. 2018, in submission) has demonstrated a vast transcriptional difference between SN and VTA astrocytes such that VTA, but not $\mathrm{SN}$, astrocytes release factors which mediate protection of VTA, SN, and induced pluripotent stem cell (iPSC) DA neurons. In addition to astrocytes, the role of microglia, another important glial cell type, in the pathogenesis of PD has become an area of great interest.

Microglia are considered to be the innate immune cells of the brain [6]. Their role in the clearance of damaged cells, such as neurons, and other foreign contaminants of the brain is well established [7-9]. It is well known that microglia derive from a myeloid lineage [10] and that injury or disease can cause increased microglial activation within the brain [11-14]. Indeed, in PD patients and animal models of $\mathrm{PD}$, greater numbers of microglia [15], as well as enhanced microglial reactivity $[16,17]$, are observed in the SN. In addition to their role as scavengers in the brain, microglia also release proand anti-inflammatory molecules in response to insult or injury $[9,18,19]$. Indeed, the canonical microglial activator lipopolysaccharide (LPS) has been used to stimulate microglial activation and the subsequent cytokine release in order to model the immune response in vivo and in vitro [12, 20-22]. Thus, it has been reported that LPS stimulation of microglia within the $\mathrm{SN}$ is sufficient to produce DA neurodegeneration [23], suggesting a role for microglia and their factors in disease progression.

Previous studies have additionally examined the interaction of microglia with PD mimetic toxins. For example, rotenone, a pesticide known to be a DA neurotoxin, was shown to require the presence of microglia in order to mediate toxicity of midbrain DA neurons in culture [24]. This raises the possibility that the interaction of PD mimetic toxins with microglia could be a potential pathway by which neurodegeneration occurs. In addition to the potential role that toxins could have on microglia, leading to an exacerbation of toxicity, the ratio of microglia to neurons within the brain could potentially produce different reactions to inflammatory stimuli. Previous work has shown that the regional distribution of microglia throughout the brain is diverse $[25,26]$, with the $\mathrm{SN}$ being populated by a high ratio of microglia to neurons. Furthermore, recent transcriptional profiling of discrete brain regions (cortex, hippocampus, cerebellum, striatum) has suggested that similar to astrocytes, microglia exhibit regionally distinct transcriptional profiles [27]. However, the potential regionality of midbrain microglia has yet to be investigated.

Therefore, in this study, we sought to determine whether midbrain SN and VTA microglia exhibit regionally specific gene expression profiles and whether these regionally isolated microglia can contribute differentially to the susceptibility of SN DA neurons to $\mathrm{MPP}^{+}$. induced toxicity as compared to the VTA. Furthermore, we investigated whether the ratio of microglia to neurons to astrocytes within the midbrain plays a role in the selective vulnerability of the $\mathrm{SN}$ to $\mathrm{PD}$ mimetic toxins. We found that regionally isolated microglia from three distinct brain regions, the cortex, SN, VTA, exhibit differential basal cytokine profiles. Furthermore, when challenged with the PD mimetic toxin $\mathrm{MPP}^{+}$, all regionally isolated microglia respond with distinct cytokine profiles. Interestingly, the addition of microglia alone is not sufficient to induce DA neuron death in cultures of either SN or VTA neurons. However, when challenged with $\mathrm{MPP}^{+}$, all regionally isolated microglia similarly exacerbate $\mathrm{MPP}^{+}$toxicity, and this exacerbation of toxicity can be alleviated by inhibiting the activation of microglia via pharmacological inhibition of the TLR4 receptor. Finally, we demonstrated that greater ratios of VTA, but not SN, astrocytes are able to protect both SN and VTA DA neurons from $\mathrm{MPP}^{+}$toxicity and that increasing the microglial ratio counters the protective effect of VTA astrocytes. Together, these results suggest that the susceptibility of SN DA neurons to a PD mimetic toxin results from a regional sensitivity to multiple environmental factors, rather than simply from an intrinsic neuronal vulnerability. A greater appreciation of the role of extrinsic mechanisms, mainly the protective nature of astrocytes and deleterious functions of microglia, in disease pathogenesis is important for the consideration of possible therapeutics in PD.

\section{Methods}

\section{Animals and IACUC policies}

All animals used in this study were maintained in accordance with the Office of Animal Resources at Thomas Jefferson University. The protocols were approved by the Institutional Animal Care and Use Committee (IACUC) at Thomas Jefferson University, protocol \#457K/01499.

\section{Embryonic tissue culture}

Timed pregnancies were performed using male and female rats from our colony of animals expressing green 
fluorescent protein under control of the human tyrosine hydroxylase promotor (TH-GFP) [1]. Embryonic day zero (E0) was determined as the first day a vaginal plug was visible. On E14.5, embryos were harvested and placed in ice cold DPBS (Gibco \#14190-144; no CaCl2 or $\mathrm{MgCl} 2$ ). GFP+ pups were visualized under a dissection microscope (Nikon SMZ1500; adjustable $\times 1-\times 11.5$ objective) by using a high-pressure mercury lamp with a 495-nm filter attachment. The midbrain was surgically extracted and cleaned of non-GFP+ tissues, and the VTA and SN were carefully separated. Tissues were collected and placed in $2.5 \mathrm{~mL}$ of enzymatic dissociation solution containing $5 \mathrm{mg}$ DNAse I (Sigma \#10104159001), $5 \mathrm{mg}$ Papain (Sigma \#10108014001), and $50 \mathrm{mg}$ L-cysteine (Sigma) for up to $30 \mathrm{~min}$. Solution was gently agitated every $5 \mathrm{~min}$ to aid in dissociation. NEP basal media (DMEM/F12, B-27 and N2 Supplements (Gibco), $1 \mathrm{mg} / \mathrm{mL}$ BSA, and Penn/Strep) was added to terminate the enzymatic reaction. Tissues were then briefly spun at $1000 \mathrm{rpm}$ for $2 \mathrm{~min}$, and the supernatant media was aspirated. Tissues were re-suspended in culture media (NEP basal media $+5 \%$ FBS) for 5 min prior to mechanical dissociation. For mechanical dissociation, a 1-mL pippetor was used with low retention tips and tissues were gently triturated eight to ten times. Cells were then pelleted for $5 \mathrm{~min}$ at $1000 \mathrm{rpm}$, re-suspended in culture media, and counted on an automated hemocytometer (Countess FLII Invitrogen). Cells were plated in a microdrop on 96-well tissue culture plates or 8 -well chamber slides pre-coated with poly-D-lysine $\left(0.5 \mathrm{mg} / \mathrm{mL}\right.$, overnight at $\left.37{ }^{\circ} \mathrm{C}\right)$ at a density of $5-7 \times 10^{4}$ cells in approximately $20 \mu \mathrm{L}$ of culture media. Cells were allowed to attach to the substrate before media was added to final volume of 100$200 \mu \mathrm{L}$ per well. Cells were allowed to mature for 6 days prior to the addition of isolated microglia (see below) in a 1:1 microglia to neuron ratio and allowed to mature for $24 \mathrm{~h}$ prior to $\mathrm{MPP}^{+}$treatment. This ratio was established based on previous studies demonstrating similar numbers of $\mathrm{MACl}+$ or $\mathrm{Iba} 1+$ microglia and $\mathrm{TH}+$ neurons within the SN [16, 28]. Experiments where inhibition of microglia was performed utilized the commercially available TLR4 antagonist 2-acetamidopyranoside (TLR4IN-C34, Sigma Cat\# SML0832). On day 7 in vitro (DIV7), C34 was added to cultures $2 \mathrm{~h}$ prior to $\mathrm{MPP}^{+}$addition. Cultures were fixed 24-48 h after $\mathrm{MPP}^{+}$treatment, and the full well was imaged using a Nikon Eclipse TI-e fitted with a Photometrics Coolsnap ES2 camera. Images were analyzed in ImageJ using a custom-designed macro for cell quantification. TH-GFP+ cell counts were compared to appropriate non-treated controls.

\section{Tissue culture-isolated astrocytes and microglia}

Astrocytes and microglia were collected using previously published methods [2-4]. Briefly, postnatal day 1-5 GFP+ pups were anesthetized on ice and quickly decapitated. Brains were removed and placed in ice cold DPBS (Gibco). GFP+ brains were visualized under a dissection microscope (Nikon SMZ1500). The midbrain and cortices were carefully dissected away from other brain regions and non-GFP+ tissues of the midbrain were cut away. The SN and VTA regions were carefully separated and collected in ice cold PBS. Whole cortices were collected and dissected into smaller pieces prior to enzymatic digestion. Tissues were then subjected to enzymatic digestion using a trypsin $(0.1 \%) /$ DNAse I $(0.5 \mathrm{mg} / \mathrm{mL})$ mixture for $5 \mathrm{~min}$ at $37^{\circ} \mathrm{C}$. The supernatant enzyme was aspirated carefully, and tissues were washed twice in DPBS. Tissues were then mechanically dissociated in $0.5 \mathrm{mg} / \mathrm{mL}$ DNAse I and gently triturated eight to ten times. Cells were then pelleted for $5 \mathrm{~min}$ at $1000 \mathrm{rpm}$, re-suspended in astrocyte media (DMEM/F12 + 10\% FBS and Penn/ Strep) and plated on tissue culture flasks. Cultures were maintained for 2-4 weeks with media changes every other day. To separate astrocytes (adherent) and microglia (non-adherent) for experimental use, flasks were shaken at $220 \mathrm{RPM}$ for $24 \mathrm{~h}$ and the non-adherent cells collected. The cell suspension was passed through a $70-\mathrm{mm}$ cell strainer prior to centrifugation at $1000 \mathrm{rpm}$ for $5 \mathrm{~min}$. The cells were re-suspended in culture media for experimental use. Samples of non-adherent cells were stained for the microglial markers Iba1 (allograft inflammatory factor 1 (Iba1, Wako \#019-19741, RRID:AB_2665520) and CD11b (BD Biosciences Cat\# 550299, RRID:AB_393594) to confirm homogeneity of the culture.

\section{Co-culture studies: astrocyte to neuron to microglia}

Neuron-astrocyte co-cultures were prepared similarly to previously published methods [5]. Briefly, isolated regional astrocytes were trypsinized, dissociated to a single cell suspension, and quantified. Increasing numbers of astrocytes $(60,000-120,000)$ were plated onto 48-well tissue culture plates. Cells were allowed to adhere and grow for $48 \mathrm{~h}$ prior to further experimentation. Subsequently, embryonic neuron preparations were carried out to obtain TH-GFP+ neurons. Neurons were plated onto regional astrocytes in a homo- and heterotypic manner at 2:1 and 4:1 (astrocyte to neuron) ratios. These ratios were established based on previous studies examining the number of astrocytes in the midbrain [28, 29], as well as our previous work demonstrating increased ratios of VTA but not SN astrocytes mediate protection from $\mathrm{MPP}^{+}$toxicity (Kostuk et al. 2018, in submission). Twenty-four hours after plating, all cultures were treated with $1 \mu \mathrm{M} \mathrm{AraC}$ to halt astrocytic proliferation. Cultures were then allowed to mature for five more days in vitro prior to microglial addition. Isolation and collection of SN and VTA microglia in sufficient numbers for 
experimental manipulation would require a vast number of animals to be sacrificed; as such, cortical microglia were used as they were easier to obtain in sufficient numbers. Microglia were added in a 1:1 and 2:1 (microglia to neuron) ratio. Twenty-four hours after microglial addition, $\mathrm{MPP}^{+}$was added at $50 \mu \mathrm{M}$ for $24-48 \mathrm{~h}$. After $\mathrm{MPP}^{+}$treatment, all cells were fixed and GFP+ cells were quantified using the Nikon Eclipse camera system described above.

\section{RNA isolation and CDNA synthesis}

Total RNA was isolated directly from freshly collected cells in TRIzol (Invitrogen), a modification of the guanidine isothiocyanate-phenol-chloroform extraction method. cDNA was synthesized by using at least $1 \mu \mathrm{g}$ total RNA in a 20- $\mu$ l reaction with Superscript IV (Invitrogen) and oligo (dT)12-18 (Invitrogen). One microliter of RNase H (Invitrogen) was added to each reaction tube, and the tubes were incubated for $20 \mathrm{~min}$ at $37^{\circ} \mathrm{C}$ before proceeding to real-time PCR.

\section{Real-time PCR analysis}

Real-time PCR was carried out on the 7500 Real Time PCR System using SYBR green PCR master mix (both from Applied Biosystems). GAPDH was used as an internal control. PCR analyses were conducted in triplicate for each sample. The reaction mix consisted of $6.35 \mathrm{ng}$ cDNA, $0.5 \mu \mathrm{M}$ forward and reverse primer mix, and $\times 1$ SYBR green PCR master mix. Reactions were run according to manufacturer protocols for at least 40 cycles. Data were analyzed using the ratiometric $\Delta \Delta C$ T method, and the mean relative mRNA expression for each sample was reported. All primer sequences used can be found in Additional file 1: Table S1.

\section{Experimental design and statistical analysis}

All data are presented as the mean \pm SEM. GraphPad was used for all statistical analysis. The statistical significance of the mean difference was calculated using a repeated measures ANOVA with appropriate post hoc analysis of significance. A $p$ value $<0.05$ was considered significant.

\section{Results}

\section{Microglia exhibit regionally specific basal cytokine profiles}

To examine the potential regionality of microglia within the midbrain, we isolated microglia from SN and VTA and compared them to microglia from cortex (CTX) using postnatal day 1-5 transgenic rats. Microglial purity was estimated at $95-100 \%$ as the vast majority of cells stained for the microglial markers Iba1 (Fig. 1a) and CD11b (Fig. 1b). Regionally isolated microglia were then collected and examined for their basal cytokine profile. We found that regionally isolated microglia exhibit subtle differences in cytokine expression profiles (Fig. 1c, d). In comparison to isolated CTX microglia (Fig. 1c, left column), SN microglia exhibit greater levels of the proinflammatory cytokines IL3, CCL3, and CCL4, while exhibiting diminished expression of IFN $\gamma$. VTA microglia (Fig. 1c, right column) conversely express greater levels of pro-inflammatory RANTES, Cxcl12, and CCL19, with decreased expression of IL10. An additional comparison was performed with CTX and SN microglia against isolated VTA microglia (Fig. 1d). We found that both SN and CTX have greatly upregulated levels of IL10 and diminished expression of CCL19 (Fig. 1d). Together, these results demonstrate a diverse regionality to microglia of the midbrain based on their baseline cytokine expression levels.

Isolated microglia have differential cytokine responses to PD mimetic toxin MPP ${ }^{+}$

Previous studies have demonstrated that in vivo treatment with the PD toxin MPTP causes microglial activation $[16,30]$. Therefore, we examined the response of our regionally isolated microglia to the active metabolite of MPTP, $\mathrm{MPP}^{+}$, to determine whether regionality results in differential activation profiles. We found that SN microglia (Fig. 2, left column) strongly upregulate the expression of pro-inflammatory IL1 $\beta$, IL $12 \alpha$, CCL3, CCL4, and CCL19, while decreasing expression of antiinflammatory IFNY in response to toxin treatment. Interestingly, while there are some similarities in the pro-inflammatory response of VTA microglia to $\mathrm{MPP}^{+}$ (Fig. 2, middle column), such as upregulation of IL1 $\beta$, CCL3, and CCL4, these microglia differ in their strong downregulation of $\mathrm{Cxcl} 12$ and significant upregulation of IL3. Finally, CTX microglia differ greatly from the other two regions examined (Fig. 2, right column). Their upregulation of pro-inflammatory cytokines is limited to TNF $\alpha$ and RANTES, but with strongly decreased expression of both IL1 $\beta$ and IL12 $\alpha$. However, CTX microglia mimic SN in their downregulation of antiinflammatory IFN $\gamma$ in response to $\mathrm{MPP}^{+}$and also decreased expression of IL6. The differential expression of cytokines in response to $\mathrm{MPP}^{+}$treatment would suggest that the addition of regionally isolated microglia would result in differential exacerbation of $\mathrm{MPP}^{+}$ toxicity of DA neurons.

\section{Regionally isolated microglia similarly exacerbate $\mathrm{MPP}^{+}$toxicity}

Due to the differences seen in the cytokine profile of regionally isolated microglia, both at baseline and in response to $\mathrm{MPP}^{+}$, we hypothesized that regionally isolated microglia would cause differential exacerbation of $\mathrm{MPP}^{+}$toxicity of midbrain DA neurons grown with their astrocytes. We find that compared to control 

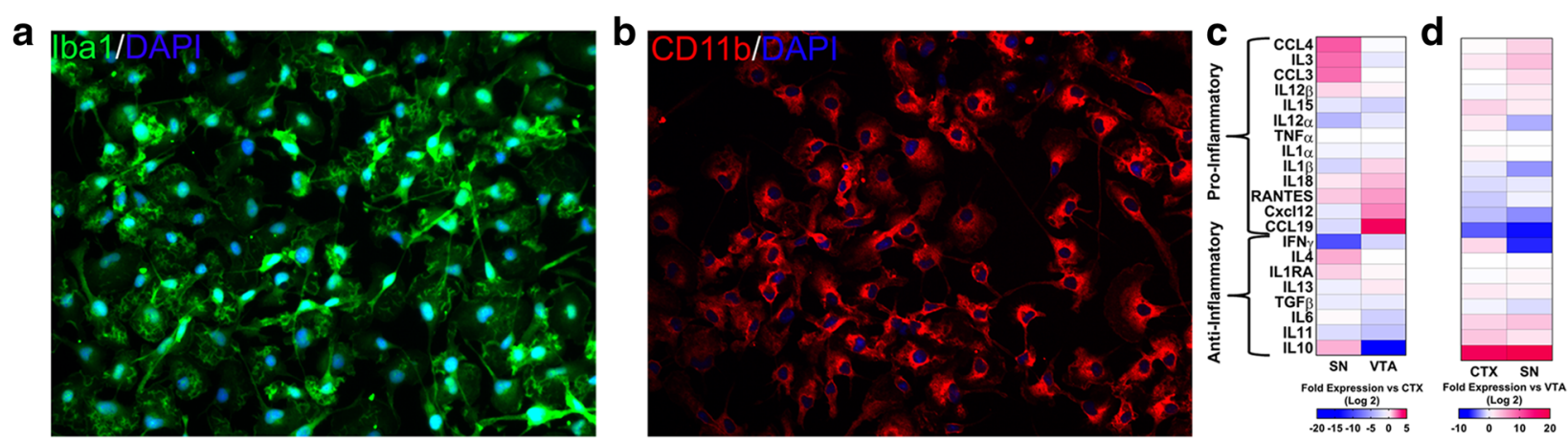

Fig. 1 Regionally isolated microglia have distinct basal cytokine profiles. Microglia from the midbrain SN and VTA as well as the cortex (CTX) were isolated based on previously established methods. We find that cultures of microglia are pure, and the vast majority (95-100\%) of cells stain for the microglial markers Iba1 (a) and CD11b (b). Regionally isolated microglia were subjected to GPCR analysis for 13 pro- and 8 anti-inflammatory cytokines and compared between different regions. In comparison to CTX microglia, SN microglia (c), left column, exhibit greater expression of the pro-inflammatory cytokines IL3, CCL3, and CCL4, with diminished IFNy expression, whereas VTA microglia (c, right column) express greater levels of RANTES, CxCl12, and CCL19, while expressing lower levels of IL10. A secondary comparison against VTA microglia (d) demonstrated that both SN (left column) and CTX (right column) microglia have greatly upregulated levels of IL10 and diminished expression of CCL19. A number of other differences in these profiles demonstrate a regional variability to microglia

cultures $(M=100$, SEM $=9.3)$, the addition of regionally isolated microglia (SN light green, $M=105$, SEM = 4.1; VTA $\tan , M=102, \mathrm{SEM}=3.1$; and CTX light purple bars, $M=105$, SEM $=3.2$ ) do not cause DA neuron degeneration in either SN (Fig. 3a) or VTA (data not shown, one-way ANOVA, $F(7,16)=1.313 p=0.3064$ ) neuron to astrocyte cultures. Interestingly, despite the regionally associated expression differences of proinflammatory cytokines upon $\mathrm{MPP}^{+}$stimulation, all regionally isolated microglia similarly exacerbate $\mathrm{MPP}^{+}$ toxicity of SN DA neurons (Fig. 3a, SN dark green, $M=$ $27, \mathrm{SEM}=1.5$; VTA orange, $M=26, \mathrm{SEM}=2.1$; and CTX dark purple bars, $M=23, \operatorname{SEM}=1.8$; one-way ANOVA with Tukey's post hoc multiple comparisons test, $\left.F(7,16)=84.2,{ }^{* * *}=p<0.001\right)$, whereas VTA DA neurons (data not shown) are robustly protected from the effect of microglia in neuron to astrocyte cultures. These results suggest that VTA, but not SN, astrocytes may be protective of their regional DA neurons despite the addition of damaging microglia regardless of their region of origin.

\section{Inhibition of microglial activation with a TLR4 antagonist prevents exacerbation of $\mathrm{MPP}^{+}$toxicity}

To confirm that microglial released factors are indeed responsible for the exacerbation of $\mathrm{MPP}^{+}$toxicity, we sought to prevent microglial activation by using a recently developed, commercially available TLR4 antagonist TLR4-IN-C34 (C34) [31]. When C34 is applied to cultures $2 \mathrm{~h}$ prior to $\mathrm{MPP}^{+}$administration, we found that C34 (Fig. 3b, light purple bar, $M=98$, SEM = 1.7) itself has no effect on the health of mixed cultures of SN neurons, astrocytes, and microglia (control, blue bar, $M=96$, SEM = 3.7). However, coupled with $\mathrm{MPP}^{+}$ treatment (Fig. 3b, dark purple, $M=56$, SEM $=1.4$ ), we demonstrated significantly decreased exacerbation of $\mathrm{MPP}^{+}$toxicity (red bar, $M=35, \mathrm{SEM}=2.1$ ) of SN DA neurons (one-way ANOVA with Tukey's post hoc multiple comparisons test, $\left.F(3,14)=144.4,{ }^{* * * * * *}=p<0.0001\right)$. Cultures where C34 inhibition has occurred resemble our previously established data where microglia are absent from cultures (Kostuk et al. 2018, in submission). This further supports that factors released from microglia indeed exacerbate $\mathrm{MPP}^{+}$toxicity of these neurons.

\section{Isolated VTA, but not SN, astrocytes mediate protection of both SN and VTA DA neurons from microglia}

As we demonstrated that VTA neurons are robustly protected from $\mathrm{MPP}^{+}$toxicity despite the presence of microglia, we sought to determine whether this protection is due to astrocytic released factors. We created both homo- and heterotypic astrocyte-neuron cultures from the SN and VTA region in varying ratios to determine if, similar to our previous findings, greater numbers of astrocytes would afford protection despite the presence of microglia. We found that in both homotypic (SN DA neurons on SN astrocytes, Fig. 4, solid blue bars: $1: 2: 1$ ratio $M=37, \mathrm{SEM}=3.4 ; 1: 4: 1$ ratio $M=34$, SEM 1.5) and heterotypic (VTA DA neurons on $\mathrm{SN}$ astrocytes, Fig. 4, solid red bars: 1:2:1 ratio $M=45, \mathrm{SEM}=2.1 ; 1: 4: 1$ ratio $M=41, \mathrm{SEM}=1.2$ ) co-cultures, higher ratios of SN astrocytes were incapable of affording greater protection of both SN and VTA neurons when microglia were present at a ratio equal to neurons. Conversely, VTA astrocytes exhibited ratio-dependent increases in protection of both $\mathrm{SN}$ and VTA DA neurons despite the presence of exacerbatory microglia in both homotypic (VTA DA 


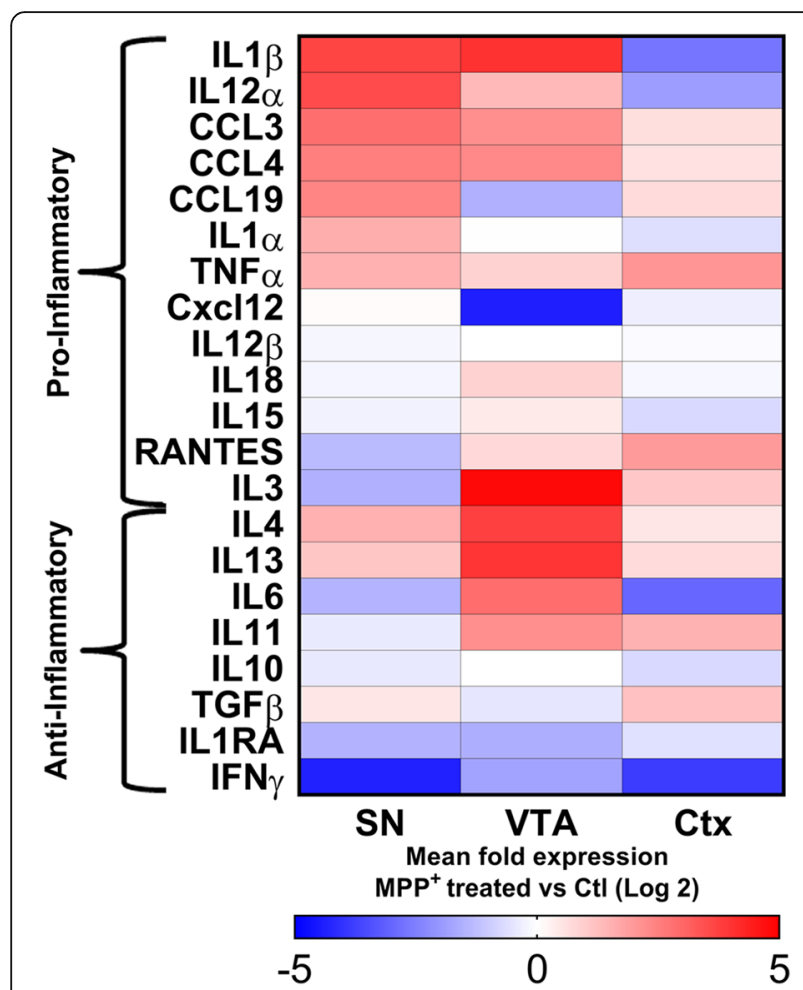

Fig. 2 Treatment of regionally isolated microglia with the Parkinson's toxin $\mathrm{MPP}^{+}$causes differential upregulation of cytokines. Regionally isolated microglia were collected and exposed to the PD toxin MPP ${ }^{+}$ for $24 \mathrm{~h}$. After toxin exposure, microglia were examined for expression of pro- and anti-inflammatory cytokines in comparison to untreated region-specific controls. MPP ${ }^{+}$-treated SN microglia (left column) exhibit a greater degree of pro-inflammatory cytokine upregulation, including IL1 $\beta$, IL12a, CCL3, CCL4, CCL19, IL1a, and TNFa, coupled with significant downregulation of IFNY compared to untreated control SN microglia. VTA microglia (middle column) share upregulation of pro-inflammatory IL1ß,CCL3, CCL4, and TNFa similar to SN microglia. However, they exhibit higher levels of IL3, as well as the anti-inflammatory cytokines IL4, IL13, and IL6. Interestingly, CTX lack upregulation of IL1 $\beta$ and IL12a, whereas they demonstrate strong expression of TNFa

neurons on VTA astrocytes, Fig. 4, hatched red bars: 1:2:1 ratio $M=82$, SEM $=1.2 ; 1: 4: 1$ ratio $M=96$, $\mathrm{SEM}=0.75$ ) and heterotypic (SN DA neurons on VTA astrocytes, Fig. 4 , hatched blue bars: $1: 2: 1$ ratio $M=86, \operatorname{SEM}=1$. 2; 1:4:1 ratio $M=89$, SEM =1.8). To further our understanding of the protection provided by VTA astrocytes, we then sought to tip the balance towards the deleterious effects of microglia by increasing their proportion of the cells in the dish. We found that the addition of microglia at a 2:1 ratio to neurons in the presence of the most protective astrocyte ratio (4:1 astrocytes to neurons) does result in DA neuron death simply because more microglia were added (data not shown). However, when coupled with $\mathrm{MPP}^{+}$ treatment, we found that the protective effect of VTA astrocytes was significantly diminished (Fig. 4; SN neurons blue vertically stripped bar, $M=57, \mathrm{SEM}=0$. 62 and VTA neurons red vertically stripped bar, $M=$ $51, \quad S E M=1$ ) by increasing the ratio of microglia (one-way ANOVA with Tukey's post hoc multiple comparisons test; $\left.F(9,28)=270.8, \quad{ }^{* * * * *} p<0.0001\right)$. These results further suggest that microglial released factors are able to overcome the protective effect of astrocytes.

\section{Discussion}

The regional vulnerability of SN DA neurons has been extensively studied for a number of years, with many studies focusing on the intrinsic underlying mechanisms and how these neurons differ from neighboring VTA neurons [2-5]. Recently, however, greater emphasis has been placed on understanding potential non-cell autonomous mechanisms, such as the impact of microglial cells, which may underlie this selective vulnerability.

In this study, we demonstrate that regionally isolated microglia of midbrain SN and VTA subregions differ in both their basal and $\mathrm{MPP}^{+}$-induced cytokine profiles. Furthermore, we demonstrate that the addition of regionally isolated microglia themselves is not deleterious to DA neurons; however, their presence significantly exacerbates $\mathrm{MPP}^{+}$toxicity in SN DA neurons. Conversely, VTA DA neurons co-cultured with astrocytes are robustly protected from $\mathrm{MPP}^{+}$toxicity despite the presence of microglia, suggesting that VTA astrocytes may be responsible for protection of these neurons. We further showed that pharmacological inhibition of microglial activation diminished the exacerbatory effect of microglia. Finally, we demonstrated that VTA astrocytes, but not $\mathrm{SN}$, are able to protect both SN and VTA DA neurons from $\mathrm{MPP}^{+}$toxicity despite the presence of deleterious microglia but that it is possible to overburden protective astrocytes with increasing numbers of microglia, thus increasing the presumptive load of microglial released factors.

The role of microglia as the immune cells of the CNS has been appreciated for a number of years [6]. Many studies have demonstrated an increased release of proinflammatory/neurotoxic species from microglia in response to inflammatory stimuli such as LPS [18]. Additionally, some studies have postulated a role for microglia in the progression of PD [11,32, 33], though a debate continues as to whether microglia play a role in initiation of disease or are activated secondary to neuronal death. Supporting the latter proposition, there are a number of reports demonstrating a response of microglia to some PD toxins. Gao et al. [24] showed that the effect of rotenone, a pesticide that has been used as a PD mimetic toxin in culture, requires the presence of microglia and that this effect is dependent on the number of microglia present within the culture. Additionally, 

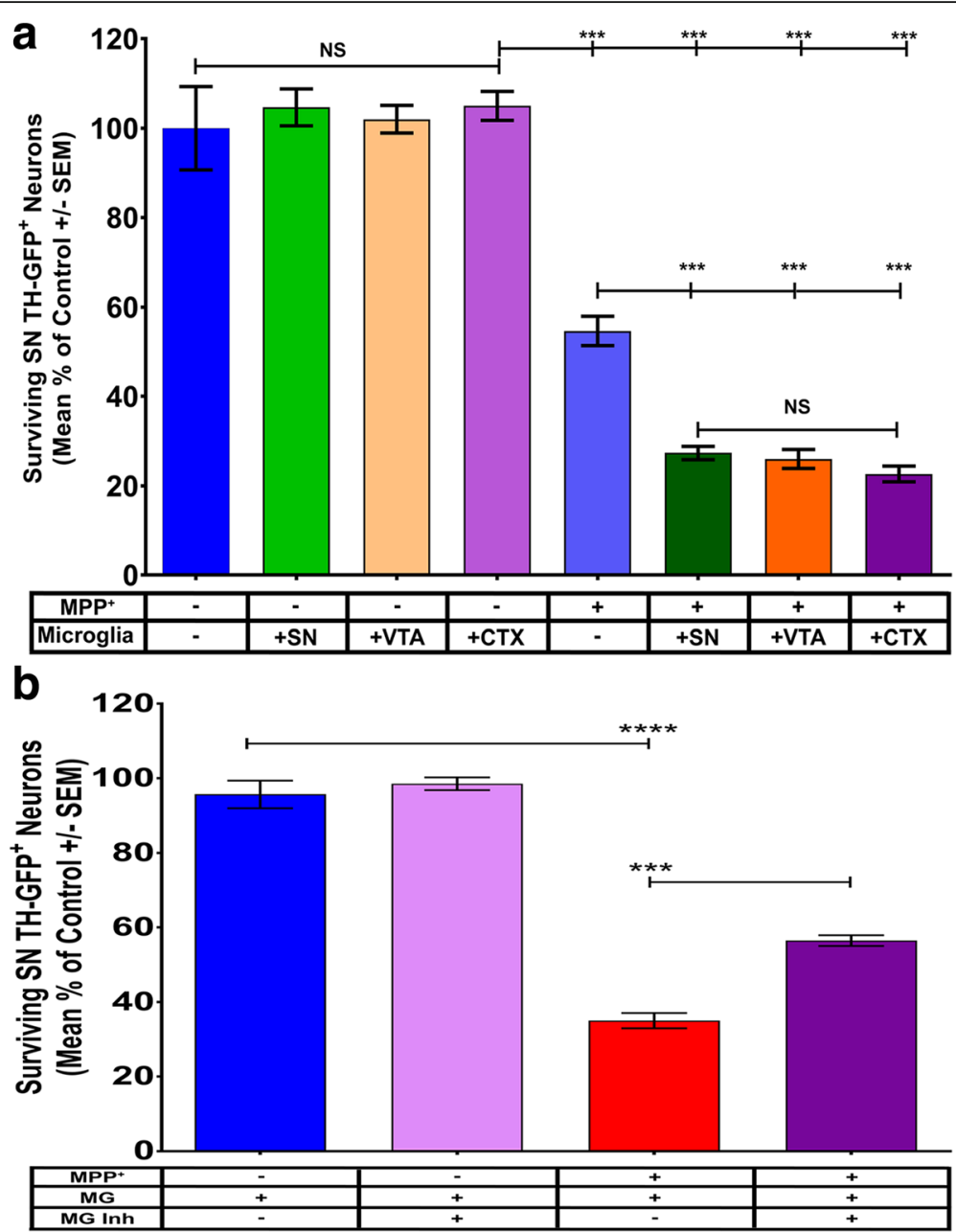

Fig. 3 Toxicity of SN DA neurons by MPP ${ }^{+}$is exacerbated in the presence of regional microglia and is alleviated by inhibition of microglial activation. a The addition of regionally isolated microglia (SN, VTA, CTX) to cultures of SN DA neurons and astrocytes demonstrates that the addition of microglia themselves is not deleterious to these neurons. However, coupled with $\mathrm{MPP}^{+}$treatment, all regionally isolated microglia cause exacerbation of toxicity to a similar extent; one-way ANOVA with Bonferroni's post hoc multiple comparisons test; $F(7,16)=84.2$,

**** $p<0.0001$. b TLR4 inhibitor C34 (light purple) does not cause any degeneration of SN DA neurons in culture. However, a significant decrease in the exacerbation of $\mathrm{MPP}^{+}$toxicity is observed when microglial activation is inhibited by the drug (red compared to purple); one-way ANOVA with Tukey's post hoc multiple comparison test; $F(3,14)=144.4{ }^{* * * *} p<0.0001$

Chien et al. [34] demonstrated that microglial released cytokines and chemokines are responsible for the loss of nigrostriatal DA neurons in response to LPS. Furthermore, recent work by Smeyne et al. [16] demonstrated that systemic injection of the PD mimetic toxin MPTP results in increased microglial reactivity within the $\mathrm{SN}$, suggesting that PD mimetic toxins can directly cause microglial activation and subsequent cytokine release. These findings are similar to ours in which microglial addition to DA neuron cultures exacerbates the effect of the $\mathrm{PD}$ mimetic toxin $\mathrm{MPP}^{+}$, suggesting that the release of pro-inflammatory molecules from microglia may have a vital role in the progression of PD DA neuron degeneration.
Previous studies have also demonstrated that the inhibition of microglial activation or knockdown of released cytokines from microglia may be neuroprotective to DA neurons. Pharmacological blockade of microglial activation [35-37] or knockdown of cytokines, such as IL-1 [38], demonstrates that prevention of microglial activation attenuates some of the degeneration seen in models of PD. Furthermore, the interaction of MPTP/ $\mathrm{MPP}^{+}$with the microglial receptor TLR4, a key receptor in the microglial activation pathway, has previously been established $[39,40]$. As such, it is unsurprising that our findings demonstrate that the use of a new TLR4 inhibitor C34 [31], prior to $\mathrm{MPP}^{+}$administration, results in decreased exacerbation of toxicity. 


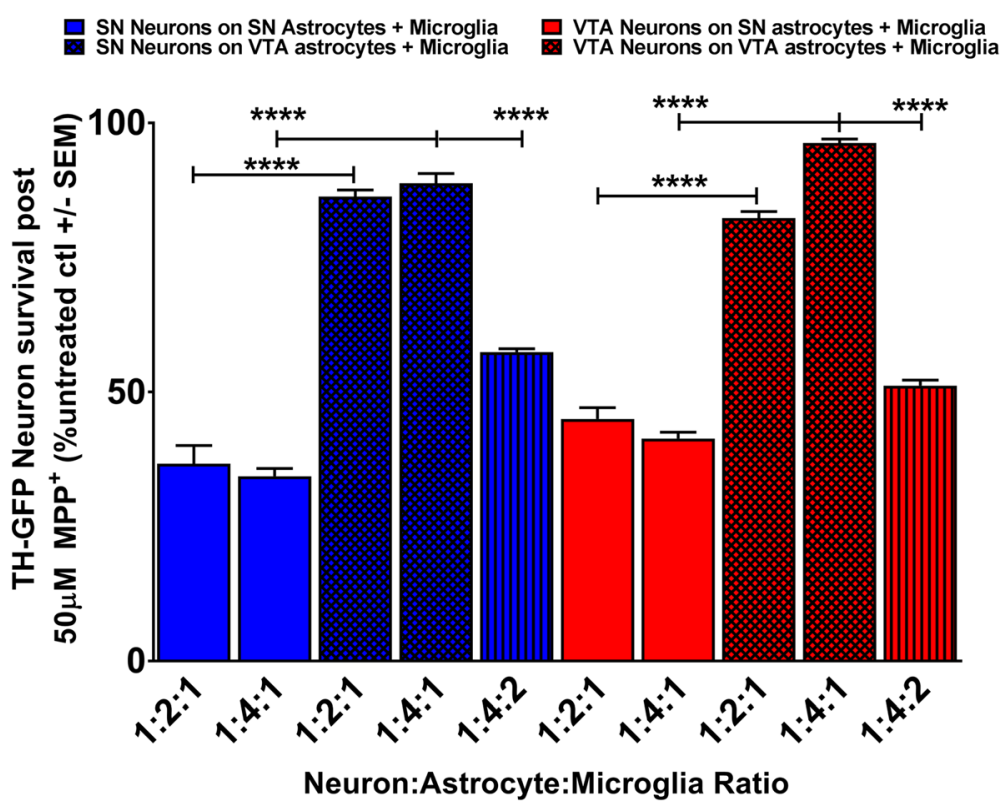

Fig. 4 Greater ratios of isolated VTA, but not SN, astrocytes protect both SN and VTA DA neurons from MPP ${ }^{+}$toxicity in the presence of microglia, but increased numbers of microglia counter this protection. Regionally homo- and heterotypic cultures were established with SN and VTA neurons/astrocytes. Greater ratios of SN astrocytes fail to increase SN (solid blue bars) or VTA (solid red bars) DA neuron survival in the presence of microglia. Conversely, VTA astrocytes robustly protect both SN (blue hatched bars) and VTA (red hatched bars) DA neurons from exacerbation of $\mathrm{MPP}^{+}$toxicity when microglia are present. The protective effect of VTA astrocytes on SN (blue vertical striped bar) and VTA (red vertical striped bar) neurons is diminished when the ratio of microglia to neurons is increased from 1:1 to 2:1. Data are presented as mean \pm SEM; one-way ANOVA with Tukey's post hoc multiple comparison test; $F(9,28)=270.8,{ }^{* * *} p<0.0001$

In addition to an effect of the PD mimetic toxins MPTP or $\mathrm{MPP}^{+}$on the activation of microglia, a number of studies have examined the impact of alpha synuclein ( $\alpha$-syn) on microglia and the subsequent activation of these cells [33, 41-43]. Indeed, studies have demonstrated that $\alpha$-syn is able to bind to surface receptors of microglia and result in their activation and subsequent upregulation of pro-inflammatory cytokines [44-46]. Therefore, it is plausible that the interaction of $\alpha$-syn and microglia of the midbrain may be exacerbating or contributing to DA neuronal degeneration similar to that seen by the effect of $\mathrm{MPP}^{+}$in our studies. However, it is still relatively unknown whether this interaction of $\alpha$-syn and microglia is an initiating factor for neuronal death or secondary to the release of species from damaged or dying neurons [43]. Furthermore, a number of studies have demonstrated that microglia can act as scavengers of extracellular $\alpha$-syn $[47,48]$, thereby clearing a potentially damaging species away from vulnerable neurons. The impact of microglial-sequestered $\alpha$-syn is not fully understood, and it is possible that internalized $\alpha$-syn could result in dysfunction of lysosomal pathways within microglia, similar to that seen in DA neurons [49], impairing normal functions within these cells. Therefore, the effect that $\alpha$-syn may have on microglia from the SN and VTA remains an important area of investigation.
The regional variability in the transcriptional profile of microglia has been investigated previously [27]. However, potential differences of microglia within the midbrain have yet to be investigated. Our study is the first of our knowledge to sub-dissect the midbrain into the vulnerable SN and protected VTA to examine the effects of isolated microglia of these regions in a PD model. Though we demonstrate a number of basal and $\mathrm{MPP}^{+}$induced cytokine differences, it is interesting to note that we fail to find any regionally specific exacerbation of $\mathrm{MPP}^{+}$toxicity. A number of possible explanations exist for this fact.

First, our investigation of the transcriptional profile of these microglia was somewhat limited. We established a list of 21 canonical pro- and anti-inflammatory cytokines based on commercially available arrays. This is a very limited view of the transcriptional profile of these cells. As such, future studies utilizing more in-depth methods of investigation, such as RNAseq, are required. These methods have been used in other models, such as spinal cord injury [50], and have demonstrated a vast array of subtle injuryinduced changes to microglia that should likewise be appreciated in the context of the midbrain in PD.

Secondly, the deleterious effect of these regionally isolated microglia could simply be due to a combined CCL3, CCL4, and TNF $\alpha$ response, as these are the three pro-inflammatory cytokines upregulated in all three 
regionally isolated microglial populations. However, the role of these cytokines has been investigated extensively in the context of PD [51-53], though the specific action of SN or VTA microglial-derived cytokines is still unknown. A possible course of investigation would be to selectively inhibit the production of these cytokines, or their downstream pathways, directly within these distinct midbrain nuclei and examine their effects in an in vivo PD model.

Finally, the response of microglia to inflammatory stimuli, such as LPS, is understood to be temporally dynamic. Indeed, previous studies have demonstrated that changes in the expression level of known pro-inflammatory cytokines can change even within $4 \mathrm{~h}$ of stimulus initiation, and these changes can be transient and disappear by $24 \mathrm{~h}$ after stimulus [54, 55]. Our examination of expression levels of cytokines was limited to the 24-h time point, as this is when we typically see the greatest death of DA neurons after $\mathrm{MPP}^{+}$treatment. However, we cannot rule out the effect of earlier cytokine responses and their impact on DA neuronal survival. Further RNAseq studies examining the temporal gene expression changes of isolated SN, VTA, and CTX microglia are required to fully understand the role these cells may have in the initiation or exacerbation of DA neuronal death in response to $\mathrm{MPP}^{+}$.

The role of astrocytes as neuroprotective cells has been well established for a number of years [56-58]. Indeed, our recent work (Kostuk et al. 2018, in submission) has demonstrated a vast transcriptional difference between the astrocytes of the SN and VTA, suggesting that VTA astrocytes are able to mediate protection of their regional DA neurons whereas $\mathrm{SN}$ astrocytes lack this ability. Furthermore, the crosstalk between astrocytes, neurons, and microglia has recently become more appreciated [59-61]. Thus, the interaction of midbrain astrocytes and microglia in the context of PD pathogenesis is an important avenue of investigation. Consequently, it is unsurprising to us that greater ratios of $\mathrm{SN}$ astrocytes fail to mediate greater protection when deleterious microglia are present and that VTA astrocytes robustly protect both SN and VTA DA neurons from $\mathrm{MPP}^{+}$toxicity despite the presence of microglia. Interestingly, however, we were able to demonstrate that it is possible to overburden protective astrocytes with greater numbers of microglia by increasing the ratio of microglia to neurons and likely their released cytokines in our cultures. This is of vital importance, as SN microglial activation and proliferation is a key feature of both human PD pathogenesis [15, 62] and animal models [16] of the disease. Therefore, as the concentration of microglial factors increases during the course of disease, less protective astrocytes in the $\mathrm{SN}$ may become more overtaxed by significant increases of microglial-released cytokines. In contrast, VTA astrocytes may be better suited to balance against these deleterious factors as there are fewer microglia and greater proportions of protective astrocytes present basally $[25,26,63,64]$, and thus, microglial activation or proliferation may never reach the critical threshold needed to cause VTA DA degeneration.

\section{Conclusions}

In summary, the data in this paper suggests that regional differences in vulnerability of midbrain DA neurons to the $\mathrm{PD}$ toxin $\mathrm{MPP}^{+}$may rely on a delicate balance between protective factors supplied by astrocytes and deleterious cytokines released by microglia. We demonstrate that regionally isolated microglia of the $\mathrm{SN}$, VTA, and CTX differ in their basal and $\mathrm{MPP}^{+}$-induced cytokine profiles. Despite these transcriptional differences, all regionally isolated microglia exacerbate MPP+ toxicity of SN DA neurons, and this exacerbation can be attenuated by inhibition of microglia activation. Furthermore, VTA, but not $\mathrm{SN}$, astrocytes are able to mediate protection from microglial-released factors, though it is possible to overcome the protective function of astrocytes with greater numbers of microglia. Thus, these findings help to expand our current view of this neurodegenerative disease process and highlight the need for greater understanding of the balanced role of astrocytes and microglia in the midbrain.

\section{Additional file}

Additional file 1: Table S1. List of primers used for qRT-PCR. (DOCX 29 kb)

\section{Abbreviations}

a-syn: Alpha synuclein; CTX: Cortex; CCL: Chemokine (C-C motif) ligand; DA: Dopaminergic; IL: Interleukin; IFNY: Interferon gamma;

LPS: Lipopolysaccharide; MPTP: 1-Methyl-4-phenyl-1,2,3,6-tetrahydropyridine; MPP': 1-Methyl-4-phenylpyridinium; PD: Parkinson's disease; SN: Substantia nigra; TNFa: Tumor necrosis factor alpha; TLR4: Toll-like receptor 4; VTA: Ventral tegmental area

\section{Funding}

This work was supported by the NIH (2 RO1 NS075839) to L.I. and the Parkinson Council.

\section{Availability of data and materials}

This manuscript contained and presented all the datasets used in the "Results" and "Conclusions" sections.

\section{Author's contributions}

EWK conceived, performed, and analyzed the experiments and wrote and edited the manuscript. JC performed the experiments and edited the manuscript. LI conceived and analyzed the experiments, wrote and edited the manuscript, and supervised and provided funding for the project. All authors approved the final version of the manuscript.

\section{Ethics approval}

All experimental procedures and animals used in this study were maintained in accordance with the Office of Animal Resources at Thomas Jefferson University. The protocols were approved by the Institutional Animal Care and Use Committee (IACUC) at Thomas Jefferson University, protocol \#457K/01499. 


\section{Competing interests}

The authors declare that they have no competing interests.

\section{Publisher's Note}

Springer Nature remains neutral with regard to jurisdictional claims in published maps and institutional affiliations.

\section{Author details}

${ }^{1}$ Department of Neuroscience, Farber Institute for Neurosciences, Thomas Jefferson University, Room 320, Bluemle Life Sciences Building, 233 S. 10th Street, Philadelphia, PA 19107, USA. ²Department of Neurology, Farber Institute for Neurosciences, Thomas Jefferson University, Room 320, Bluemle Life Sciences Building, 233 S. 10th Street, Philadelphia, PA 19107, USA. ${ }^{3}$ Department of Neurosurgery, Farber Institute for Neurosciences, Thomas Jefferson University, Room 320, Bluemle Life Sciences Building, 233 S. 10th Street, Philadelphia, PA 19107, USA.

Received: 14 February 2018 Accepted: 27 April 2018

Published online: 11 May 2018

\section{References}

1. Hirsch E, Graybiel AM, Agid YA. Melanized dopaminergic neurons are differentially susceptible to degeneration in Parkinson's disease. Nature. 1988:334:345-8

2. Chung $C Y$, Seo H, Sonntag KC, Brooks A, Lin L, Isacson O. Cell type-specific gene expression of midbrain dopaminergic neurons reveals molecules involved in their vulnerability and protection. Hum Mol Genet. 2005;14:1709-25. https://doi.org/10.1093/hmg/ddi178.

3. Phani S, Gonye G, lacovitti L. VTA neurons show a potentially protective transcriptional response to MPTP. Brain Res. 2010;1343:1-13. https://doi.org/10.1016/j.brainres.2010.04.061

4. Thuret S, Bhatt L, O'Leary DDM, Simon HH. Identification and developmental analysis of genes expressed by dopaminergic neurons of the substantia nigra pars compacta. Mol Cell Neurosci. 2004;25:394-405. https://doi.org/10.1016/j.mcn.2003.11.004.

5. Yao F, Yu F, Gong L, Taube D, Rao DD, Mackenzie RG. Microarray analysis of fluoro-gold labeled rat dopamine neurons harvested by laser capture microdissection. J Neurosci Methods. 2005;143:95-106.

6. Streit WJ. Microglia as neuroprotective, immunocompetent cells of the CNS. Glia. 2002;40(2):133-9.

7. Neher JJ, Neniskyte U, Brown GC. Primary phagocytosis of neurons by inflamed microglia: potential roles in neurodegeneration. Front Pharmacol. 2012;3:27. https://doi.org/10.3389/fphar.2012.00027.

8. Wang Y, Cella M, Mallinson K, Ulrich JD, Young KL, Robinette ML, et al. TREM2 lipid sensing sustains the microglial response in an Alzheimer's disease model. Cell. 2015;160:1061-71. https://doi.org/10.1016/j.cell.2015.01.049.

9. Neumann H, Kotter MR, Franklin RJM. Debris clearance by microglia: an essential link between degeneration and regeneration. Brain. 2009;132(Pt 2):288-95. https://doi.org/10.1093/brain/awn109.

10. Alliot F, Godin I, Pessac B. Microglia derive from progenitors, originating from the yolk sac, and which proliferate in the brain. Brain Res Dev Brain Res. 1999;117:145-52. http://www.ncbi.nlm.nih.gov/pubmed/ 10567732

11. Kim YS, Joh TH. Microglia, major player in the brain inflammation: their roles in the pathogenesis of Parkinson's disease. Exp Mol Med. 2006;38:333-47.

12. Liu B, Hong J-S. Role of microglia in inflammation-mediated neurodegenerative diseases: mechanisms and strategies for therapeutic intervention. J Pharmacol Exp Ther. 2003;304:1-7.

13. Loane DJ, Byrnes KR. Role of microglia in neurotrauma. Neurotherapeutics. 2010;7:366-77.

14. Weinstein JR, Möller T. Microglia in ischemic brain injury. Future Neurol. 2010;5:227-46.

15. McGeer PL, Itagaki S, Boyes BE, McGeer EG. Reactive microglia are positive for HLA-DR in the substantia nigra of Parkinson's and Alzheimer's disease brains. Neurology. 1988;38:1285-91. https://doi.org/10.1212/WNL.38.8.1285.

16. Smeyne RJ, Breckenridge CB, Beck M, Jiao Y, Butt MT, Wolf JC, et al. Assessment of the effects of MPTP and paraquat on dopaminergic neurons and microglia in the substantia nigra pars compacta of C57BL/6 mice. PLoS One. 2016;11:e0164094. https://doi.org/10.1371/journal.pone.0164094.
17. Joers V, Tansey MG, Mulas G, Carta AR. Microglial phenotypes in Parkinson's disease and animal models of the disease. Prog Neurobiol. 2017;155:57-75. https://doi.org/10.1016/j.pneurobio.2016.04.006.

18. Hanisch UK. Microglia as a source and target of cytokines. Glia. 2002;40:140-55.

19. Smith JA, Das A, Ray SK, Banik NL. Role of pro-inflammatory cytokines released from microglia in neurodegenerative diseases. Brain Res Bull. 2012;87:10-20. https://doi.org/10.1016/j.brainresbull.2011.10.004.

20. Appel K, Honegger P, Gebicke-Haerter PJ. Expression of interleukin-3 and tumor necrosis factor-beta mRNAs in cultured microglia. J Neuroimmunol. 1995;60:83-91. http://www.ncbi.nlm.nih.gov/pubmed/7642751

21. Hoogland ICM, Houbolt C, van Westerloo DJ, van Gool WA, van de Beek D. Systemic inflammation and microglial activation: systematic review of animal experiments. J Neuroinflammation. 2015;12:114. https://doi.org/10. 1186/s12974-015-0332-6

22. Buttini $M$, Boddeke $H$. Peripheral lipopolysaccharide stimulation induces interleukin-1 beta messenger RNA in rat brain microglial cells. Neuroscience. 1995;65:523-30. http://www.ncbi.n/m.nih.gov/pubmed/7777165

23. Gao HM, Jiang J, Wilson B, Zhang W, Hong JS, Liu B. Microglial activationmediated delayed and progressive degeneration of rat nigral dopaminergic neurons: relevance to Parkinson's disease. J Neurochem. 2002;81:1285-97.

24. Gao H-M, Hong J-S, Zhang W, Liu B. Distinct role for microglia in rotenone-induced degeneration of dopaminergic neurons. J Neurosci. 2002:22:782-90

25. Lawson LJ, Perry VH, Dri P, Gordon S. Heterogeneity in the distribution and morphology of microglia in the normal adult mouse brain. Neuroscience. 1990;39(I):151-70

26. Kim WG, Mohney RP, Wilson B, Jeohn GH, Liu B, Hong JS. Regional difference in susceptibility to lipopolysaccharide-induced neurotoxicity in the rat brain: role of microglia. J Neurosci. 2000;20:6309-16.

27. Grabert K, Michoel T, Karavolos MH, Clohisey S, Baillie JK, Stevens MP, et al. Microglial brain region-dependent diversity and selective regional sensitivities to aging. Nat Neurosci. 2016;19:504-16. https://doi.org/10.1038/ $\mathrm{nn} .4222$.

28. Smeyne M, Jiao Y, Shepherd KR, Smeyne RJ. Glia cell number modulates sensitivity to MPTP in mice. Glia. 2005;52(2):144-52

29. Smeyne M, Goloubeva O, Smeyne RJ. Strain-dependent susceptibility to MPTP and MPP+-induced parkinsonism is determined by glia. Glia. 2000:2001(34):73-80

30. Yasuda Y, Shimoda T, Uno K, Tateishi N, Furuya S, Yagi K, et al. The effects of MPTP on the activation of microglia/astrocytes and cytokine/chemokine levels in different mice strains. J Neuroimmunol. 2008;204:43-51. https://doi.org/10.1016/j.jneuroim.2008.08.003.

31. Neal MD, Jia H, Eyer B, Good M, Guerriero CJ, Sodhi CP, et al. Discovery and validation of a new class of small molecule toll-like receptor 4 (TLR4) inhibitors. PLoS One. 2013;8:1-10.

32. Moehle MS, West a B. M1 and M2 immune activation in Parkinson's disease: foe and ally? Neuroscience. 2014; https://doi.org/10.1016/j.neuroscience. 2014.11.018.

33. Sanchez-Guajardo $\mathrm{V}$, Tentillier N, Romero-Ramos $\mathrm{M}$. The relation between asynuclein and microglia in Parkinson's disease: recent developments. Neuroscience. 2015;302:47-58. https://doi.org/10.1016/j.neuroscience.2015. 02.008 .

34. Chien $\mathrm{CH}$, Lee MJ, Liou HC, Liou HH, Fu WM. Microglia-derived cytokines/ chemokines are involved in the enhancement of LPS-induced loss of nigrostriatal dopaminergic neurons in DJ-1 knockout mice. PLoS One. 2016;11:1-24.

35. Liu B, Du L, Hong JS. Naloxone protects rat dopaminergic neurons against inflammatory damage through inhibition of microglia activation and superoxide generation. J Pharmacol Exp Ther. 2000;293:607-17.

36. Borrajo A, Rodriguez-Perez Al, Villar-Cheda B, Guerra MJ, Labandeira-Garcia $J \mathrm{~L}$. Inhibition of the microglial response is essential for the neuroprotective effects of rho-kinase inhibitors on MPTP-induced dopaminergic cell death. Neuropharmacology. 2014;85:1-8.

37. Wu DC, Jackson-Lewis V, Vila M, Tieu K, Teismann $P$, Vadseth $C$, et al. Blockade of microglial activation is neuroprotective in the 1- methyl-4phenyl-1,2,3,6-tetrahydropyridine mouse model of Parkinson disease. J Neurosci. 2002;22:1763-71.

38. Tanaka S, Ishii A, Ohtaki H, Shioda S, Yoshida T, Numazawa S. Activation of microglia induces symptoms of Parkinson's disease in wild-type, but not in IL-1 knockout mice. J Neuroinflammation. 2013;10:143. https://doi.org/10. 1186/1742-2094-10-143. 
39. Noelker C, Morel L, Lescot T, Osterloh A, Alvarez-Fischer D, Breloer M, et al. Toll like receptor 4 mediates cell death in a mouse MPTP model of Parkinson disease. Sci Rep. 2013;3:1393. https://doi.org/10.1038/srep01393.

40. Zhou P, Weng R, Chen Z, Wang R, Zou J, Liu X, et al. TLR4 signaling in $\mathrm{MPP}^{+}$-induced activation of BV-2 cells. Neural Plast. 2016;2016:5076740. https://doi.org/10.1155/2016/5076740.

41. Béraud D, Hathaway HA, Trecki J, Chasovskikh S, Johnson DA, Johnson JA, et al. Microglial activation and antioxidant responses induced by the Parkinson's disease protein a-synuclein. J Neurolmmune Pharmacol. 2013;8:94-117. https://doi.org/10.1007/s11481-012-9401-0.

42. Sekiyama K, Sugama S, Fujita M, Sekigawa A, Takamatsu Y, Waragai M, et al. Neuroinflammation in Parkinson's disease and related disorders: a lesson from genetically manipulated mouse models of a-synucleinopathies. Parkinsons Dis. 2012;2012

43. Schapansky J, Nardozzi JD, LaVoie MJ. The complex relationships between microglia, alpha-synuclein, and LRRK2 in Parkinson's disease. Neuroscience. 2015;302:74-88. https://doi.org/10.1016/j.neuroscience.2014.09.049.

44. Kim C, Ho D-H, Suk J-E, You S, Michael S, Kang J, et al. Neuron-released oligomeric a-synuclein is an endogenous agonist of TLR2 for paracrine activation of microglia. Nat Commun. 2013;4:1562. https://doi.org/10.1038/ ncomms 2534

45. Harms AS, Cao S, Rowse AL, Thome AD, Li X, Mangieri LR, et al. MHCII is required for a-synuclein-induced activation of microglia, CD4 T cell proliferation, and dopaminergic neurodegeneration. J Neurosci. 2013;33: 9592-600. https://doi.org/10.1523/JNEUROSCI.5610-12.2013.

46. Ihse E, Yamakado H, van Wijk XM, Lawrence R, Esko JD, Masliah E. Cellular internalization of alpha-synuclein aggregates by cell surface heparan sulfate depends on aggregate conformation and cell type. Sci Rep. 2017;7:9008. https://doi.org/10.1038/s41598-017-08720-5.

47. Rey NL, Petit GH, Bousset L, Melki R, Brundin P. Transfer of human asynuclein from the olfactory bulb to interconnected brain regions in mice. Acta Neuropathol. 2013;126:555-73. https://doi.org/10.1007/s00401-0131160-3.

48. Lee H-J, Cho E-D, Lee KW, Kim J-H, Cho S-G, Lee S-J. Autophagic failure promotes the exocytosis and intercellular transfer of a-synuclein. Exp Mol Med. 2013;45:e22. https://doi.org/10.1038/emm.2013.45.

49. Mazzulli JR, Zunke F, Isacson O, Studer L, Krainc D. a-Synuclein-induced lysosomal dysfunction occurs through disruptions in protein trafficking in human midbrain synucleinopathy models. Proc Natl Acad Sci U S A. 2016; 113:1931-6. https://doi.org/10.1073/pnas.1520335113.

50. Noristani HN, Gerber YN, Sabourin J-C, Le Corre M, Lonjon N, MestreFrances $\mathrm{N}$, et al. RNA-Seq analysis of microglia reveals time-dependent activation of specific genetic programs following spinal cord injury. Front Mol Neurosci. 2017;10:90. https://doi.org/10.3389/fnmol.2017.00090.

51. Ray A, Sehgal N, Karunakaran S, Rangarajan G, Ravindranath V. MPTP activates ASK1-p38 MAPK signaling pathway through TNF-dependent Trx1 oxidation in parkinsonism mouse model. Free Radic Biol Med. 2015;87:312-25. https:/doi.org/10.1016/j.freeradbiomed.2015.06.041.

52. Zhu Y, Chen X, Liu Z, Peng Y-P, Qiu Y-H. Interleukin-10 protection against lipopolysaccharide-induced neuro-inflammation and neurotoxicity in ventral mesencephalic cultures. Int J Mol Sci. 2015;17 https://doi.org/10.3390/ ijms17010025.

53. Mogi M, Harada M, Riederer $P$, Narabayashi $H$, Fujita K, Nagatsu T. Tumor necrosis factor-alpha (TNF-alpha) increases both in the brain and in the cerebrospinal fluid from parkinsonian patients. Neurosci Lett. 1994;165:208-10. https://doi.org/10.1016/0304-3940(94)90746-3.

54. Lund S, Christensen KV, Hedtjärn M, Mortensen AL, Hagberg H, Falsig J, et al. The dynamics of the LPS triggered inflammatory response of murine microglia under different culture and in vivo conditions. J Neuroimmunol. 2006;180:71-87

55. Norden DM, Trojanowski PJ, Villanueva E, Navarro E, Godbout JP. Sequential activation of microglia and astrocyte cytokine expression precedes increased Iba-1 or GFAP immunoreactivity following systemic immune challenge. Glia. 2016;64:300-16. https://doi.org/10.1002/glia.22930.

56. Bélanger M, Magistretti PJ. The role of astroglia in neuroprotection. Dialogues Clin Neurosci. 2009;11:281-96.

57. Allen NJ, Barres BA. Neuroscience: glia—more than just brain glue. Nature. 2009;457 February:675-677

58. Barres BA. The mystery and magic of glia: a perspective on their roles in health and disease. Neuron. 2008;60:430-40. https://doi.org/10.1016/j. neuron.2008.10.013.
59. Correa FG, Hernangómez M, Guaza C. Understanding microglia-neuron cross talk: relevance of the microglia-neuron cocultures. Methods Mol Biol. 2013;1041:215-29. https://doi.org/10.1007/978-1-62703-520-0_20.

60. Liu W, Tang Y, Feng J. Cross talk between activation of microglia and astrocytes in pathological conditions in the central nervous system. Life Sci. 2011;89:141-6. https://doi.org/10.1016/j.lfs.2011.05.011.

61. Lian H, Litvinchuk A, Chiang AC-A, Aithmitti N, Jankowsky JL, Zheng H. Astrocyte-microglia cross talk through complement activation modulates amyloid pathology in mouse models of Alzheimer's disease. J Neurosci. 2016;36:577-89. https://doi.org/10.1523/JNEUROSCI.2117-15.2016.

62. Perry VH. Innate inflammation in Parkinson's disease. Cold Spring Harb Perspect Med. 2012;2:a009373. https://doi.org/10.1101/cshperspect.a009373.

63. Nair-Roberts RG, Chatelain-Badie SD, Benson E, White-Cooper H, Bolam JP, Ungless MA. Stereological estimates of dopaminergic, GABAergic and glutamatergic neurons in the ventral tegmental area, substantia nigra and retrorubral field in the rat. Neuroscience. 2008;152:1024-31. https://doi.org/ 10.1016/j.neuroscience.2008.01.046

64. Damier P, Hirsch EC, Zhang P, Agio Y. Glutathione peroxidase, glial cells and Parkinson's disease. Neuroscience. 1993;52:1-6.

\section{Ready to submit your research? Choose BMC and benefit from:}

- fast, convenient online submission

- thorough peer review by experienced researchers in your field

- rapid publication on acceptance

- support for research data, including large and complex data types

- gold Open Access which fosters wider collaboration and increased citations

- maximum visibility for your research: over $100 \mathrm{M}$ website views per year

At BMC, research is always in progress.

Learn more biomedcentral.com/submissions 